\title{
Naturally Derived Nanomaterials for Multidisciplinary Applications and Beyond
}

\author{
Yue Zhang ${ }^{1, *}$ and Yangchao Luo ${ }^{2, *}$ \\ Received: 10 June 2021; Accepted: 23 June 2020. \\ Article type: Editorial article.
}

\begin{abstract}
Nanomaterials are a popular subject in the food, environment, medical and safety research, which applications hold promising future in practical perspective. ${ }^{[1]}$ The increasing demand for nanomaterials with high quality and safety, and concerns of environment sustainable development have been encouraging researchers to exploit novel, green biodegradable nanostructures from natural resource, many of which could be identified as food and naturally derived nanomaterials. ${ }^{[2]}$ Compared with synthesized polymers, food derived nanomaterials have low human health and safety risks, and are environmentally friendly. However, they may contain multicomponents with more complex structures, and are spatially inhomogeneous and polydisperse, which bring challenges along with new opportunities for their practical applications. Therefore, in this fourth issue in ES Food \& Agroforestry, we have selected a few papers focusing on recent and novel applications of naturally drived materials in multidisciplinary fields.
\end{abstract}

Prasad et al. reviewed recent applications of nanotechnology in various forms in the area of veterinary medicine. Authors also recommended to conduct more works at the cellular and molecular levels to address the concerns from public about potential risks of using these nanostructures. Li et al. introduced an emerging usage of cellulose, one of the most abundant natural biopolymers, in the development of flexible sensors and possible applications as electronic skin, wearable device and medical equipment. There is no doubt that remarkable progress on cellulose flexible sensing materials will be achieved in near future.

Water pollution is an increasing global concern, which

School of Food Science and Biotechnology Zhejiang Gongshang University Hangzhou, China, 310018.

Department of Nutritional Sciences, University of Connecticut, Storrs, CT 06269, USA.

*Email: zhangyue@zjgsu.edu.cn (Y. Zhang), yangchao.luo@uconn.edu (Y. Luo) requires effective and low-cost treatments to deal with waste water. In this collection, we have included one research paper from Sun et al. by introducing a promising approach of using corncob-derived activated carbon for effective removal of organic pollutants in sewage. The porous nanostructures developed by biomass materials could be a solution to treat environmental pollutants.

In addition to above review and research papers, Fleming and Luo have initiated a very insightful discussion on the fact of the dietary patterns and lifestyles people have been taking during national lockdowns might against the recommendations for preventing morbidity and mortality of COVID-19. The authors also provided another way of looking at the impact of COVID-19 lockdown.

As editors, we would like to thank all authors and reviewers for their valuable contributions. We hope that the collections in this issue could inspire more technologies and applications from peers on naturally-derived materials, as well as more discussions on this pandemic in the view of food and nutrition scientists.

\section{References}

[1] M. Miernicki, T. Hofmann, I. Eisenberger, F. von der Kammer, A. Praetorius, Nat. Nanotechnol., 2019, 14, 208-216, doi: 10.1038/s41565-019-0396-z.

[2] Y. Huang, L. Mei, X. Chen, Q. Wang, Nanomaterials, 2018, 8, 830 , doi: $10.3390 /$ nano8100830.

\section{Author information}

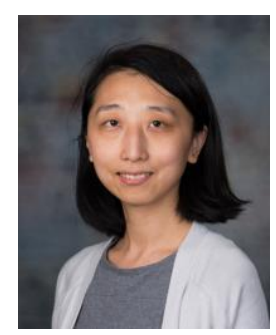

Yue Zhang is a Professor in the School of Food Science and Biotechnology at Zhejiang Gongshang University, China. Dr. Zhang obtained her B.S in Chemistry and Ph.D in Physical Chemistry from Wuhan University (2002 2011). She worked as a postdoc research assoc iate at the 
University of Tennessee in 2011 2015, and then worked as an Assistant Professor at the University of Nebraska Lincoln prior to joining Zhejiang Gongshang University in 2019. Dr. Zhang's research mainly focuses on the physicochemical properties of food biopolymers and the development of biopolymer based nano formulations for targeted delivery purpose.

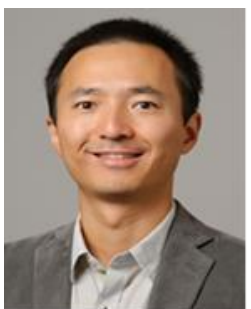

Yangchao Luo is an Associate Professor in the Department of Nutritional Sciences at the University of Connecticut. Dr. Luo's research is highly interdisciplinary and his laboratory applies materials science and engineering principles to understand physical and chemical interactions among natural biomaterials at the nanoscale and design novel nanodelivery systems for applications in food safety, quality, and functionality

Publisher's Note: Engineered Science Publisher remains neutral with regard to jurisdictional claims in published maps and institutional affiliations. 